\title{
State of the Art of Palytoxin and Analogs Analytical Methods for Seafood Monitoring
}

\author{
Vitor Vasconcelos ${ }^{1,2}$, Joana Azevedo ${ }^{2,3}$, Marisa Silva ${ }^{1,2}$, Vitor Ramos 1,2
}

Review: Palytoxin group (PLTXs) are produced by marine organisms, like zoanthids (Palythoa) and benthic dinoflagellates (Ostreopsis). Besides originally found in tropical regions, reports on the presence of PLTXs in subtropical and temperate marine waters have grown considerably in the past decade. This is related to the increase of Ostreopsis spp. blooms in such environments, which pose considerable risk to the human health. In fact, contamination of seafood with Ostreopsis spp. already caused human poisoning incidents after the ingestion of clupeoid fish, some with fatal outcomes [1,2]. Therefore there is an urgent need to establish regulations on PLTX-group toxins in seafood intended for human consumption. Due to lack of exposure assessment and analytical information about these toxins, authorities are not able to establish a reliable guideline limit. European Food Safety Authority (EFSA) gives an estimated value for palytoxin and ostreocin-D of $30 \mu \mathrm{g} / \mathrm{Kg}$ shellfish meat [1]. Chemically these natural products are large and complex molecules, having both hydrophilic and lipophilic behaviors consisting of 64 chiral centers, 40-42 hydroxyl and 2 amide groups. These characteristics confer their heat resistance, water solubility and a large number of possible analogs. The analytical approach to identify and quantify PLTXs, such as HPCE-UV, HPLC-UV and FLD or LC-MS, it's mainly chosen depending on the type of matrix and on the limit of detection required. The most sensitive method for Ostreopsis cultures, having a limit of detection (LOD) of $0.75 \mathrm{ng}$ on column [3,4], implies the derivatization of the molecule and until know was not validated for seafood intended for human consumption. In order to better monitor this emergent phenomenon there is a need to develop techniques and to produce certificated standards and reference materials to achieve proper method validation, following International Conference on Harmonization (ICH) and Food and Drug Administration (FDA) guidelines. This work overviews the actually available methods to analyze PLTXs - highlighting their strengths, limitations and adequacy for seafood monitoring, while it points out possible analytical requirements and future approaches.

\section{References}

EFSA Panel on Contaminants in the Food Chain (CONTAM), 2009. The EFSA Journal 1306, 1-23. Available from: www. efsa.europa.eu (December 2010).

Ramos, V., Vasconcelos, V., 2010. Mar. Drugs 8, 2021-2037. Rióbo, P., Paz, B., V., Franco J. M., 2006. Anal. Chim. Acta 566, 217-223.

Rióbo, P., Franco J. M., 2011. Toxicon. 57, 368-375.

Financial Support: This project is partially financed by the interreg project PHARMATLANTIC and the FCT project PTDC/MAR/099642/2008.

\footnotetext{
${ }^{1}$ Department of Biology, Faculty of Sciences, Porto University, Portugal

2 Marine and Environmental Research Center - CIIMAR/CIMAR Porto University, Portugal

${ }^{3}$ School of Health Technology of Porto, Vila Nova de Gaia, Portugal E-mail address: vmvascon@fc.up.pt (V. Vasconcelos).
} 